\title{
Emotion Differentiation and Bipolar Risk in Emerging Adults Before and During the COVID-19 Pandemic
}

Cynthia M. Villanueva ${ }^{1}$, Stevi G. Ibonie ${ }^{1}$, Emily Jensen ${ }^{2,3}$, Lucca Eloy ${ }^{2,3}$, Jordi Quoidbach ${ }^{4}$, Angela Bryan ${ }^{1}$, Sidney D’Mello²,3, \& June Gruber ${ }^{1}$

${ }^{1}$ Department of Psychology and Neuroscience, University of Colorado Boulder

${ }^{2}$ Institute of Cognitive Science, University of Colorado Boulder

${ }^{3}$ Department of Computer Science, University of Colorado Boulder

${ }^{4}$ Department of People Management and Organisation, Universitat Ramon Llull, ESADE, Barcelona, Spain

Corresponding Authors:

Cynthia M. Villanueva and June Gruber

Department of Psychology and Neuroscience

University of Colorado Boulder

345 UCB, Muenzinger D318

Boulder, CO 80309-0345 USA

Email: cynthia.villanueva@colorado.edu and june.gruber@colorado.edu 


\begin{abstract}
Despite the prominence of emotion disturbance in bipolar disorder, few studies have assessed emotion differentiation. The present investigation used an experience-sampling approach to test the utility of emotion differentiation in predicting bipolar mood-related difficulties. Across two studies, emerging adults participated during a normative first year of college (Spring 2019; Study $1 ; n=136)$ or during their first year of college marked by a naturalistic global pandemic stressor (Spring 2020; Study 2; $n=136$ ). Study 1 results suggested that emotion differentiation was not associated with trait bipolar risk. Study 2 suggested that global emotion differentiation was associated with increased trait bipolar risk, but not current mood symptom severity. These results suggest that relationships between emotion differentiation and dimensions of bipolar risk may vary by context. Discussion focuses on the implications for translational interventions.
\end{abstract}

Keywords: emotion differentiation, emotion dynamics, bipolar risk, mania, emerging adults, college, COVID-19, experience sampling 


\section{Emotion Differentiation and Bipolar Risk in Emerging Adults Before and During the COVID-19 Pandemic: An Experience-Sampling Approach}

Bipolar disorder is a chronic and severe affective disorder, characterized by periods of expansive and elevated positive mood (mania or hypomania) and frequently also by periods of depression including dysphoric mood or loss of pleasure (e.g., American Psychiatric Association, 2013a). Bipolar disorder is associated with significant and persistent impairments in social and occupational domains of functioning (e.g., Judd et al., 2002), increases risk for other psychiatric and medical conditions, and is associated with a strikingly high suicide rate (e.g., Fagiolini et al., 2013). The onset of bipolar mood episodes coincides with a vulnerable lifespan period in young adulthood (e.g., Kennedy et al., 2005) known as emerging adulthood (Arnett, 2007), characterized by increased risk of mental health difficulties (e.g., Auerbach et al., 2016; Bruffaerts et al., 2018) and often overlaps with heightened experiences of social, financial, and academic stressors common during college. Given the staggering costs of bipolar disorder and heightened risk for onset during emerging adulthood (Chandler et al., 2008; Kennedy et al., 2005; Merikangas et al., 2011), it is critical to improve our understanding of potential factors which may help identify risk and precede the onset of bipolar disorder. Disrupted emotion processes have been underscored as key processes involved in bipolar disorder (e.g., Alloy et al., 2009; Gruber, 2011; Johnson et al., 2007; Phillips \& Vieta, 2007), and are thus a promising avenue for further investigation.

\section{Emotion-Related Disturbances in Bipolar Disorder}

Emotion-related difficulties are a hallmark feature of bipolar disorder. A growing body of work suggests that bipolar disorder is characterized by disruptions in emotion reactivity and regulation. With respect to emotion reactivity, defined as the magnitude of change in emotion 
response from a baseline state (Gross \& Jazaieri, 2014), laboratory studies utilizing standardized (e.g., films; Gruber et al., 2008; Gruber, Harvey et al., 2011), idiographic (e.g., autobiographical memory; Gruber et al., 2009), and social (e.g., dyadic interactions; Dutra et al., 2014) stimuli suggest that risk for and diagnosis of bipolar disorder is associated with elevated and sustained positive emotion responding across contexts in response to positive, negative, and neutral stimuli (for a brief review, see Gruber, 2011). By contrast, few to no differences in negative emotion responding emerged comparing bipolar disorder with non-psychiatric healthy controls (e.g., Gruber, 2011; Johnson et al., 2007). Other work assessing momentary emotions outside of the laboratory using experience-sampling approaches shows decreased positive emotions in individuals at risk or diagnosed with bipolar disorder (or no differences) compared to healthy controls (e.g., Gruber et al., 2013; Myin-Germeys et al., 2003), as well as increased levels of negative emotions in daily life compared to controls, even during periods of remission (e.g., Gruber et al., 2013; Sperry \& Kwapil, 2019). With respect to emotion regulation, defined as the process by which individuals modify their emotions (Gross \& Jazaieri, 2014), research suggests that bipolar disorder is associated with difficulties regulating both positive and negative emotions (e.g., Gruber, Eidelman et al., 2011; Johnson et al., 2008) and utilizing ineffective regulation strategies in the laboratory (e.g., Gruber et al., 2012) and in daily life (Gruber et al., 2013). Taken together, this research suggests that troubled emotion functioning is central to bipolar disorder. Yet it remains less well understood what factors may be maintaining these emotionrelated disturbances in bipolar prone and diagnosed samples.

\section{Emotion Differentiation as a Window into Bipolar Disorder}

We argue that one understudied but central process that may contribute to and maintain emotion-related difficulties in bipolar disorder is emotion differentiation, the ability to identify 
and experience one's emotions with specificity (e.g., Pond et al., 2012; Smidt \& Suvak, 2015). Individuals can vary in their degree of emotion differentiation based on the extent to which they make fine-grained distinctions between similar emotions across both valence (e.g., positive versus negative) and arousal (e.g., low versus high) dimensions of emotions (e.g., Barrett, 1998; Feldman, 1995). For example, a person who experiences anger, annoyance, and anxiousness as separate distinct emotions in response to different events would have higher emotion differentiation than both a person who consistently experiences all three emotions in response to the same situations, and a person who experiences simply feeling bad or unpleasant, representing emotions in more global negative terms with less specificity. Importantly, high emotion differentiation may be achieved through different routes. A person who tends to experience a single emotion at a time (e.g., excitement) would have higher emotion differentiation than a person who experiences a mix of different emotions (e.g., excitement, happiness, contentment). However, a person who experiences multiple emotions at the same time can also have high emotion differentiation, provided that different situations give rise to unique combinations of emotions at varying intensities (even if emotions sometimes co-occur; Nook et al., 2018). Increased emotion differentiation has been hypothesized to convey critical information about different situations, sources of emotions, and possible courses of action that allow for more flexible and adaptive emotion regulation responses (Kashdan et al., 2015; Kalokerinos et al., 2019; Thompson et al., 2021).

Variation in emotion differentiation (or ED hereafter) has been linked to psychological and health outcomes. For example, higher emotion differentiation is associated with useful coping behaviors — such as more thorough information processing before acting — and greater resilience to stressors (e.g., Tugade et al., 2004), as well as more effective emotion regulation 
skills and strategies when dealing with intense emotions (e.g., Kalokerinos et al., 2019; O’Toole et al., 2014). ED is also associated with fewer maladaptive responses to emotions, such as lower likelihood to engage in binge drinking when stressed (Kashdan et al., 2010) or to respond aggressively when angered (Pond et al., 2012). Furthermore, interventions that train individuals to better differentiate their emotions result in distress reductions (e.g., Kircanski et al., 2012). By contrast, low levels of ED have been observed across a range of emotion-related disorders, such as depression (e.g., Demiralp et al., 2012; Liu et al., 2019; Willroth et al., 2019), anxiety (e.g., Kashdan \& Farmer, 2014), autism (e.g., Erbas et al., 2013), borderline personality disorder (e.g., Suvak et al., 2011; Tomko et al., 2015), and anorexia-related eating disorders (e.g., Selby et al., 2014). Moreover, lower levels of ED have been associated with poorer clinical outcomes, such as higher rates of non-suicidal self-injury in patients with borderline personality disorder (Zaki et al., 2013), greater likelihood of initial relapse among substance users (Anand et al., 2017), and more weight-loss behaviors in individuals with anorexia (Selby et al., 2014). With respect to mood disorders, specifically, lower levels of emotion differentiation have been associated with elevated depression (e.g., Willroth et al., 2019). In one study, depressed individuals had lower negative emotion differentiation than healthy controls in a one-week experience-sampling study (Demiralp et al., 2012). Among adults with depressive symptoms, ED has also been demonstrated to moderate associations between stressful life events and rumination (Liu et al., 2019; Nook et al., 2020; Starr et al., 2017, 2019). Taken together, a growing body of research suggests that diminished ED has been associated both cross-sectionally and longitudinally with clinically relevant mood and related outcomes.

\section{The Present Investigation}


The present investigation is the first to our knowledge to examine ED in emerging adults at risk for bipolar disorder. We examined ED in relationship to bipolar relevant mood outcomes during a critical socioemotional transition period in emerging adults' lives: the first year of college. For traditional age college students, this is where the median age of onset for bipolar disorder commonly occurs and challenges to emotion responding are also common. We examined ED using an ecologically valid two-week experience-sampling approach. ED was assessed during a typical period of transition to college in Study 1, and a context in which stressors were amplified during the naturalistic socioemotional stressor posed by the Coronavirus 2019 (COVID-19) pandemic in Study 2. This allowed us to examine the unique importance of stressors in examining links between ED and bipolar mood difficulties. Lastly, we assessed participants' abilities to differentiate both negative and positive emotions ${ }^{1}$ which enabled us to more carefully examine nuanced ED dimensions. ${ }^{2}$ We predicted that bipolar risk would be associated with decreased ED generally and across both positive and negative ED subscales. We also examined whether these hypothesized patterns of results were consistent across both studies (i.e., pre-COVID and COVID acute outbreak). We ensured that any findings obtained held when accounting for emotion intensity and symptom severity.

\section{Study 1: Experience-Sampling Approach to Emotion Differentiation and Bipolar Risk}

Study 1 assessed the relationship between emotion differentiation and individual differences using a well-validated measure of bipolar risk severity in an emerging adult sample during their first year of college using an ecologically valid and rigorous experience-sampling design.

\section{Method}




\section{Participants}

Participants were 136 American emerging adults during their first year of college recruited as part of a larger study on emotion and mental health in emerging adults at the University of Colorado Boulder (IRB \#18-0483). Participants were initially recruited via posted flyers, online advertisements, and email advertisements distributed to full-time first-year college students during the 2018-2019 academic year at the University of Colorado Boulder. Inclusion criteria included being a self-reported full-time first-year college student at CU Boulder between the ages of 18-25, fluent in English, and having completed usable datasets for the primary demographic and clinical study measures described below. ${ }^{3}$ See Table 1 for descriptives. Supplementary materials can be found at https://github.com/junegruber/villanueva

\section{Bipolar Disorder Risk}

Bipolar disorder risk was assessed using a validated 20-item version of the Hypomanic Personality Scale (HPS-20; Meads \& Bentall, 2008; Sperry et al., 2015). The HPS-20 is a trait measure of bipolar disorder risk consisting of a self-report true/false questionnaire assessing trait-like bipolar-relevant changes in mood (e.g., "I often feel excited and happy for no apparent reason"), energy ("I often have moods where I feel so energetic and optimistic that I feel I could outperform almost anyone at anything"), and cognition ("Sometimes ideas and insights come to me so fast I cannot express them all”) with higher scores reflecting increased risk for bipolar disorder. Previous work has documented that the HPS has good predictive validity for the prospective onset of manic and hypomanic mood episodes (e.g., Eckblad \& Chapman, 1986; Kwapil et al., 2000; Meyer \& Hautzinger, 2003). As there is no established clinical cutoff score for the HPS-20 and we were interested in examining continuous bipolar disorder risk, scores 
were treated as a continuous variable in our regression models. Internal consistency for the HPS20 was $\operatorname{good}(a=0.75)$.

\section{Bipolar Disorder Mood Symptoms}

Current symptoms of mania and depression, a common part of the diagnostic criteria for bipolar disorders, were self-reported using the Diagnostic and Statistical Manual of Mental Disorders Fifth Edition Cross-Cutting Symptom Measure (DSM5-CCSM; American Psychiatric Association, 2013b). The DSM5-CCSM scale is a 23-item self-report measure of psychiatric symptoms rated over the past two weeks covering 13 distinct psychiatric dimensions. Each item is rated on a 0 (none) to 4 (severe) scale indicating how much each symptom has bothered the individual for the past two weeks. The present investigation focused specifically on the depression and mania symptom domains. The depression domain consisted of two items assessing anhedonia ("little interest or pleasure in doing things") and dysphoria or sad mood ("feeling down, depressed or hopeless"). The mania domain consisted of two items assessing both hyperactivity ( "starting lots more projects than usual or doing more risky things than usual") and reduced sleep ("sleeping less than usual, but still have a lot of energy?”). Depression (i.e., DSM5-Depression) and mania (i.e., DSM5-Mania) symptoms were scored using the maximum or highest value following scoring recommendations. Following scoring guidelines, we additionally assessed mania severity using the Altman Self Rating Mania Scale (ASRM; Altman et al., 1997), a 5-item self-report scale assessing current symptoms of mania over the past week. Items were rated on a 0 to 4 scale with total scores computed as the sum across the individual items with greater scores indicating increased mania symptom severity, with clinically significant mania symptom cutoff scores of $\geq 14$ ( $n=4$ scored above this cutoff). Internal consistency was adequate $(a=0.67)$. 


\section{Experience-Sampling Measures}

Participants completed an experience-sampling procedure using the ExpiWell smartphone-based application (previously Expimetrics) downloaded to their personal smartphone (https://app.expiwell.com). Specifically, participants completed four brief quasi-random surveys between 9am-9pm (i.e., 9:00 a.m. - 12:00 p.m., 12:00 - 3:00 p.m., 3:00 - 6:00 p.m., and 6:00 9:00 p.m.) for approximately 2 weeks for a total of up to 60 experience-sampling events. Participants were prompted to fill out the questionnaires within 15 minutes of receiving a notification, after which time the current survey would deactivate and no longer accept responses. Questions included current emotions, thoughts, and the context or activities in which the prompt occurred. At the end of the ESM data collection period, participants who completed at least $50 \%$ of prompts were compensated $\$ 40$ and participants who completed more than $85 \%$ were awarded an additional bonus of $\$ 10$, for a total of $\$ 50$. Participants who completed less than $50 \%$ of entries were not included in data analysis $(n=31)$. There were no significant differences in any of the clinical characteristics between participants who completed less than $50 \%$ of entries and participants who were retained in analyses for completing at least $50 \%$ of ESM entries ( $p s>.05)$. However, compared to completers, non-completers (i.e., $<50 \%$ ESM entries) had significantly higher mean socioeconomic status $(M=7.35, S D=1.23$ versus $M=6.71$, $S D=1.43)$. Participants included in the analysis completed an average of $M=44.03$ total trials $(S D=7.28)$ with a range of $30-58$ trials. With respect to emotion experience, participants selfreported their current experience of 12 emotion items commonly used for momentary assessment studies (Kahneman et al., 2004). Participants rated how they felt right now for distinct positive and negative emotions rated on a 7-point Likert scale from 0 (not at all) to 6 (very much). These 
emotion items were used to compute ED and control for positive and negative emotion intensity (i.e., PA and NA).

ED. Following previous conventions (e.g., Demiralp et al., 2012; Smidt \& Suvak, 2015), individual emotion items were used to compute an index of ED. We computed ED separately for negative ED (using the 6 negative emotion items, i.e., frustrated/annoyed, depressed/blue, hassled/pushed around, angry/hostile, worried/anxious, criticized/put down; referred to as EDNegative), positive ED (using the 3 positive emotion items, i.e., happy, warm/friendly, enjoying myself; referred to as ED-Positive), and general ED (modeled after Liu et al., 2019 as the sum of negative ED + positive ED; referred to as ED-General). Following Kahneman and colleagues’ (2004) computations, we excluded three items (i.e., tired, impatient for it to end, competent/capable) that were not directly measuring emotion concepts. From this, ED-General, ED-Positive, and ED-Negative were computed using intra-class correlation coefficients (ICCs; absolute agreement; Thompson et al., 2021; Tugade et al., 2004) between emotion terms across the sampling period, which provided a measure of how ratings for different emotions covary, or change in tandem, across the ESM data collection period. In line with previous ED studies, ICCs were then Fisher $z$-transformed and subtracted from 1, such that higher scores indicated increased ED or the tendency for participants to rate emotions differently (versus similarly) across multiple time points. ${ }^{4}$

In line with prior research that has demonstrated consistent correlations between ED and emotion intensity, we controlled for mean PA and NA intensity scores in all analyses to ensure observed effects were independent of emotion intensity level (c.f., Thompson et al., 2021). PA and NA emotional intensity scores were calculated by averaging across the ratings for all the time points (i.e., up to 60 total) of the sampling period individually for each of the emotion items 
(i.e., a mean score was first computed separately for each of the 6 negative emotion items and 3 positive emotion items). We then computed composite PA and NA scores by averaging across the mean scores for each of the positive emotion items (i.e., to create the mean PA composite) and averaging across the mean scores for each of the negative emotion items (i.e., to create the mean NA composite). Higher scores indicate higher mean PA and NA intensity, respectively. PA ranged from 0.35-5.72 $(M=3.33, \mathrm{SD}=1.02)$. NA ranged from 0.06-3.65 $(M=1.18, S D=0.78)$.

\section{Procedure}

Participants completed a cross-sectional Qualtrics survey in either the Fall 2018 or Spring 2019 semester lasting approximately 60 minutes which included the HPS-20, current clinical symptoms (i.e., ASRM, DSM5-Depression, DSM5-Mania) and other measures not part of the present investigation; (See Supplementary Materials). Participants were compensated via cash (\$20), Amazon gift card (\$20), or course credit (2 credits). Participants who consented in the survey to be recontacted were followed up at the end of the Spring 2019 semester to participate in a follow-up survey to re-collect current symptom information again (for Fall 2018 participants) and the ESM study. Experience-sampling data collection was completed for all participants during the end of the first year of college which was an average of $M=29.68$ $(S D=13.41)$ days after completion of the survey administered during Spring 2019 which contained self-reported current clinical symptoms.

\section{Study 1 Results}

\section{Preliminary Analyses}

We first examined the distribution of variables included in the analyses. None of our variables significantly departed from normality based on these indices (i.e., skewness $+/-2$ and kurtosis +/-7; Hair et al., 2016). As seen in Table 2, ED-General was correlated with increased 
ED-Positive and ED-Negative. Consistent with past research, there was a moderate positive correlation between ED-Positive and ED-Negative (e.g., Boden et al., 2013), and ED-General and ED-Negative were both correlated with decreased NA. Of note, ED-Positive was correlated with increased ASRM. Correlations between clinical measures and emotion intensity were in the expected directions. No other correlations were significant.

\section{Study 1 Main Analyses: ED and Bipolar Disorder Risk}

To examine the relationship between ED and bipolar disorder risk, we used a hierarchical linear regression analysis. Specifically, two separate regressions were run with bipolar disorder risk (HPS-20) as the outcome, which was done first for global ED (ED-General) and separately for the positive and negative ED indices (ED-Positive and ED-Negative). Block 1 included age and gender (Male=0, Female=1), Block 2 included PA and NA intensity, and Block 3 included ED-General or ED-Positive and ED-Negative, respectively. No significant outliers were revealed with Cook's distance and multicollinearity diagnostics suggested satisfactory tolerance statistics, and missing cases were deleted listwise. This allowed us to examine the influence of ED-General and positive and negative ED subscale scores on bipolar disorder risk while accounting for common confounds. ${ }^{5}$

We first examined whether the ED-General score was a predictor of trait bipolar disorder risk. As seen in Table 3, the demographic variables in Block 1 were not associated with bipolar disorder risk scores using the HPS-20 (Model 1: $F_{(2,110)}=0.87, p=.421 ; R^{2}=.02$ ). When current PA and NA intensity were added in Block 2, the overall model was still not significant (Model 2: $\left.F_{(4,108)}=1.06, p=.378 ; R^{2}=.04, \Delta R^{2}=.02\right)$. The overall model for Block 3 was not significant (Model 3: $F_{(5,107)}=0.84, p=.522 ; R^{2}=.04, \Delta R^{2}=.00$ ). These results remained largely consistent 
when controlling for current mood symptoms (see Supplementary Materials). ${ }^{6}$ Taken together, this suggests that ED-General did not predict trait bipolar disorder risk.

We next examined whether the ED-Positive and ED-Negative valence scores were predictors of trait bipolar disorder risk. As seen in Table 4, the demographic variables in Block 1 were not predictors of bipolar disorder risk scores using the HPS-20 (Model 1: $F_{(2,110)}=0.87$, $p=.421 ; R^{2}=.02$ ). When current PA and NA intensity were added in Block 2 , the overall model was still not significant (Model 2: $F_{(4,108)}=1.06, p=.378 ; R^{2}=.04, \Delta R^{2}=.02$ ). Inconsistent with our hypotheses, the overall model for Block 3 was not significant (Model 3: $F_{(6,106)}=0.78, p=.591$; $R^{2}=.04, \Delta R^{2}=.00$ ). These results remained consistent when controlling for current mood symptoms (see Footnote 6 and Supplementary Materials). Taken together, this suggests that EDPositive and ED-Negative did not predict trait bipolar disorder risk.

\section{Study 1 Brief Discussion}

Contrary to our predictions, ED did not predict trait bipolar risk in Study 1. However, preliminary bivariate correlations suggested that some measures of ED may track elevations in state-based measures of bipolar disorder-relevant mood symptomatology. Specifically, EDPositive was associated with increased mania symptoms (i.e., ASRM), a finding that was distinct from more general measures of ED or negative ED. However, Study 1 should be interpreted with the following limitations in mind. First, fewer emotion items were used to compute positive ED than to compute negative ED. This was due to using an existing emotion scale (DRM; Kahneman et al., 2004) that was not originally designed to optimize measures of ED. Second, although we collected symptoms of depression and mania, these measures were assessed approximately one month prior to the first day of experience-sampling. Thus, we were unable to determine whether any associations between ED and dimensions of bipolar disorder were state-dependent, due to 
current mood symptomatology that was not captured. Third, while experience-sampling measures of ED have the advantage of assessing ED in peoples' everyday lives and are a common method of measuring the construct, a potential issue is a lack of emotionally rich experiences present during the sampling period, which could severely restrict computations of ED. Timing the experience-sampling period to capture a range of emotional experiences and intensities would optimize our ability to detect potential links with bipolar risk dimensions. We tried to address these limitations in Study 2.

\section{Study 2: Experience-Sampling Approach to ED and Bipolar Risk Dimensions During an}

\section{Acute Stressor}

Study 2 was designed to test the relationship between ED and bipolar risk dimensions in emerging adults in their first year of college during a period of heightened socioemotional stress. Based on preliminary correlational analyses from Study 1, we broadened our conceptualization of bipolar disorder risk to include both a longer-term dispositional or trait-like indicator of bipolar risk (i.e., HPS-20) as well as more state-based measures of current bipolar disorder mania and depression mood symptoms (i.e., DSM5-Mania, ASRM, and DSM5-Depression). To capture ED during a period of heightened variability of emotional experiences while enduring an unprecedented stressor, uncertainty, and social isolation, we employed parallel experiencesampling methods from Study 1 during the Coronavirus 2019 (COVID-19) pandemic outbreak near the end of the Spring 2020 semester. The COVID-19 acute outbreak in 2020 was characterized by unprecedented social isolation and preventative health measures to slow the spread of COVID-19 infection. Governmental policies calling for adherence to self-quarantine resulted in disruption of lives and work, skyrocketing levels of unemployment and economic 
instability, and university suspension of on-campus activities. Recent research has emphasized the need to understand and address heightened risk of college student mental health challenges during the COVID-19 pandemic (e.g., Cao et al., 2020; Taquet et al., 2021; Wang et al., 2020). Moreover, prior research has suggested the particular importance of differentiating emotions in the context of stressful events (e.g., Nook et al., 2020; Starr et al., 2019). Study 2 hence provided an unusual opportunity to examine how ED may function differently in the context of an unexpected naturally occurring and collective socioemotional stressor. Study 2 also included additional self-report emotion items to widen the repertoire and sensitivity of our ED measures and included additional current clinical symptoms of mania and depression closer in time to the experience-sampling period (i.e., on average within the week prior to ESM data collection), which allowed us to more precisely investigate whether ED measures were associated with more comprehensive trait-like and state-based dimensions of bipolar disorder risk.

\section{Method}

\section{Participants}

Similar to Study 1, participants were a distinct American sample of 136 first-year college students between the ages of 18-25 during the 2019-2020 academic year at the University of Colorado Boulder (IRB \#18-0483). Participants were recruited using a similar approach as Study 1. Inclusion criteria were the same as in Study $1 .{ }^{7}$ Given the purported rise in mental health concerns during the COVID-19 pandemic, we note that participants in Study 2 reported greater difficulty with depression symptoms, lower self-reported levels of mania symptom severity, and reported lower PA intensity than Study 1 participants. See Table 1 for characteristics and scale descriptives.

\section{Bipolar Disorder Risk}


We used the same measure of trait bipolar disorder risk (HPS-20; Meads \& Bentall, 2008) as Study 1. Internal consistency in Study 2 was good $(a=0.73)$.

\section{Bipolar Disorder Mood Symptoms}

Current depression and mania symptoms were assessed using the same measures as described in Study 1 (i.e., DSM5-Depression, DSM5-Mania, and ASRM). Study 2 had additional measures of continuous depression symptoms using the Beck Depressive Inventory-Short Form scale (BDI-SF; Beck \& Beck, 1972), a 13-item self-report inventory of depressive symptoms in the past week, rated on a scale of 0 to 3 with greater summed scores indicating increased depression severity in the past week. Approximately $20 \%(n=27)$ scored above recommended depression symptom cutoffs (e.g., Furlanetto et al., 2005). Internal consistency in Study 2 was good for the BDI-SF $(a=0.89)$ though lower for the ASRM $(a=0.53){ }^{8}$

\section{Experience-Sampling Measures}

Study 2 used a parallel experience-sampling method as in Study 1, lasting approximately 2 weeks during the end of participants' first year of college coinciding with the acute COVID-19 outbreak when lockdown restrictions were in place (i.e., between April-May 2020). Experiencesampling questions included identical items as in Study 1 with the addition of several positive and negative emotion items described below. Also consistent with Study 1 procedures, participants who completed at least $50 \%$ of total ESM prompts (i.e., data were rendered usable) were compensated via a $\$ 40$ Amazon gift card. Those who completed more than $85 \%$ were awarded an additional bonus of $\$ 10$, for a total of $\$ 50$. There were no significant differences in demographics or clinical characteristics between participants who completed less than $50 \%$ of entries and participants who were retained in analyses for completing at least $50 \%$ of ESM 
entries. Eligible participants completed an average of $M=43.40$ total trials $(S D=6.58)$ with a range of $28-53$.

Consistent with Study 1, current self-reported positive and negative emotions were assessed four times a day during the experience-sampling period (Kahneman et al., 2004). Two positive and one negative emotion item (i.e., grateful/thankful, optimistic/hopeful, and lonely) were added to more broadly sample the valence and arousal dimensions of emotion and to capture common and context-appropriate affective experiences such as loneliness during the acute COVID-19 pandemic outbreak. Self-reported emotion ratings were used to compute an index of ED using the same approach as Study 1 (see Footnote 4), with the additional one negative item (lonely) and two additional positive items (grateful/thankful, optimistic/hopeful). We computed mean PA and NA intensity scores as in Study 1, with the additional items noted. PA ranged from 0.02-5.92 $(M=2.79, \mathrm{SD}=1.30)$. NA ranged from 0.05-4.49 $(M=1.01, S D=0.74)$. As in Study 1, we included PA and NA as covariates in our main analyses of ED.

\section{Procedure}

Similar to Study 1, participants completed a cross-sectional Qualtrics survey in either the Fall 2019 or Spring 2020 semester lasting approximately 60 minutes which included the HPS-20, current clinical symptoms (i.e., ASRM, DSM5-Mania, BDI-SF, DSM5-Depression) and other measures not part of the present investigation; (See Supplementary Materials). Participants were compensated via cash (\$20), Amazon gift card (\$20), or course credit (2 credits). Participants who consented in the survey to be recontacted were followed up at the end of the Spring 2020 semester to participate in a follow-up survey to re-collect current symptom information again (for all participants) and the ESM study. Experience-sampling data collection was completed for all participants during the end of the first year of college which was an average of $M=7.56$ 
$(S D=6.25)$ days after completion of the Spring 2020 survey which contained self-reported current clinical symptoms.

\section{Study 2 Results}

\section{Preliminary Analyses}

We first examined the distribution of our primary variables of interest. None of our variables significantly departed from normality (though see Footnote 8 for ASRM score distribution). As seen in Table 5, we examined bivariate correlations between our primary variables. In contrast with Study 1, lower ED-General and ED-Negative were associated with increased trait bipolar risk (HPS-20) and increased depressive symptoms (BDI-SF). Lower EDNegative was also associated with increased difficulty with mania symptoms (DSM5-Mania). Similar to Study 1, trait bipolar disorder risk was associated with difficulty with mania symptoms (DSM5-Mania), higher ED-Negative was associated with decreased NA, ED-General correlated with increased ED-Positive and ED-Negative and decreased NA, and ED-Positive and ED-Negative were positively correlated with one another. Similar to Study 1, manic symptoms (ASRM and DSM5-Mania) were associated with decreased depressive symptoms (BDI-SF and DSM5-Depression) and increased PA, and depressive symptoms (BDI-SF and DSM5Depression) were associated with decreased PA and increased NA. HPS-20 was also associated with increased depressive symptoms (BDI-SF) and increased PA and NA. Difficulty with mania symptoms (DSM5-Mania) was associated with increased mania severity (ASRM) and increased difficulty with depressive symptoms (DSM5-Depression). Difficulty with depressive symptoms (DSM5-Depression) were associated with increased depressive symptom severity (BDI-SF), and depressive symptoms (BDI-SF, DSM5-Depression) were associated with increased NA and decreased PA. No other correlations were significant. 


\section{Study 2 Main Analyses: ED and Trait Bipolar Disorder Risk}

Study 2 utilized a similar regression analysis approach to Study 1 (see Footnote 5). Two separate regressions were run where trait bipolar disorder risk (HPS-20) was the outcome, first for global ED (ED-General) and separately for the positive and negative ED indices (EDPositive, ED-Negative). Block 1 included relevant confounds of age and gender (Male=0, Female=1), Block 2 included mean PA and NA intensity, and Block 3 included either EDGeneral or ED-Positive and ED-Negative for our first and second regression models, respectively. No significant outliers were revealed with Cook's distance, multicollinearity diagnostics suggested satisfactory tolerance statistics, and missing cases were deleted listwise.

We first examined whether the ED-General score was a predictor of trait bipolar disorder risk in Study 2. As seen in Table 3, the demographic variables in Block 1 were not associated with HPS-20 (Model 1: $\left.F_{(2,118)}=0.34, p=.716 ; R^{2}=.01\right)$. When current PA and NA intensity were added in Block 2, the overall model became significant (Model 2: $F_{(4,116)}=4.23, p=.003 ; R^{2}=.13$, $\Delta R^{2}=.12$ ); notably, both PA and NA intensity were associated with increased HPS-20. Consistent with our hypotheses, when ED-General was added in Block 3, the overall model was significant (Model 3: $F_{(5,115)}=4.45, p=.001 ; R^{2}=.16, \Delta R^{2}=.03$ ), and examination of individual beta weights indicated that ED-General was associated with decreased HPS-20 scores risk $(\beta=-0.19, p=.033)$. As predicted, lower ED was associated with increased trait bipolar disorder risk. We note that this pattern remained consistent when controlling for current symptoms (see Footnote 6 and Supplementary Materials).

We next examined whether the ED-Positive and ED-Negative valence scores were predictors of trait bipolar disorder risk in Study 2. As seen in Table 4, the demographic variables in Block 1 were not associated with bipolar disorder risk scores using the HPS-20 (Model 1: $F_{(2}$, 
$\left.{ }_{118)}=0.34, p=.716 ; R^{2}=.01\right)$. When current PA and NA intensity were added in Block 2 , the overall model was significant (Model 2: $F_{(4,116)}=4.27, p=.003 ; R^{2}=.13, \Delta R^{2}=.12$ ); notably, both PA and NA intensity were associated with increased bipolar risk scores. When ED-Positive and ED-Negative were added to Block 3, the overall model was significant (Model 3: $F_{(6,114)}=3.68$, $\left.p=.002 ; R^{2}=.16, \Delta R^{2}=.03\right)$. However, examination of the individual beta weights indicated that neither ED-Positive $(\beta=-0.10, p=.300)$ nor ED-Negative $(\beta=-0.14, p=.171)$ alone predicted bipolar disorder risk scores and the $\Delta F$ from Model 2 to Model 3 was also not significant $(p=.103)$. We note that this pattern of findings remained consistent when controlling for current mood symptoms (see Footnote 6 and Supplementary Materials). Contrary to our predictions, these results indicate that ED-Positive and ED-Negative subscales did not predict trait bipolar disorder risk.

\section{Study 2 Main Analyses: ED and Bipolar Disorder Symptom Dimensions}

We examined whether ED was associated with our bipolar disorder-relevant mania and depression symptom dimensions in Study 2 more formally. These analyses paralleled our main analyses but included our symptom measures (DSM5-Mania, ASRM, DSM5-Depression, BDISF) as the outcome in place of trait bipolar disorder risk (HPS-20). ${ }^{9}$ We first examined whether the ED-General score was a predictor of symptom measures in Study 2. Four separate regressions were run for each of the two depression and two mania outcome measures. Block 1 included relevant confounds of age and gender, Block 2 included mean PA and NA intensity, and Block 3 included ED-General. The demographic variables in Block 1 did not predict any of the depression or mania measures (Model 1: $p \mathrm{~s}>.20$ ). When PA and NA intensity were added in Block 2, the overall model was significant for DSM-5 Depression (Model 2: $F_{(4,109)}=9.04$, $\left.p<.001 ; R^{2}=.25, \Delta R^{2}=.23\right)$ and BDI-SF (Model 2: $\left.F_{(4,109)}=19.59, p<.001 ; R^{2}=.42, \Delta R^{2}=.40\right)$, and 
the DSM-5 Mania measure (Model 2: $F_{(4,109)}=2.50, p=.047 ; R^{2}=.08, \Delta R^{2}=.06$ ), but not the ASRM (Model 2: $\left.F_{(4,110)}=2.29, p=.064 ; R^{2}=.8, \Delta R^{2}=.07\right)$. Specifically, NA intensity predicted increased depression symptoms on the BDI-SF and difficulties associated with depression symptoms on the DSM5-Depression measure. Of note, NA intensity also predicted increased mania symptoms on the DSM5-Mania measure, and PA predicted increased mania symptoms on the ASRM measure and decreased depression symptoms on the BDI-SF. The overall models for Block 3 remained significant for depression symptoms on the BDI-SF $\left(\right.$ Model 3: $F_{(5,108)}=7.22$, $p<.001 ; R^{2}=.25, \Delta R^{2}=.00$ ) and DSM-5 Depression measure (Model 3: $F_{(5,108)}=15.54, p<.001$; $\left.R^{2}=.42, \Delta R^{2}=.00\right)$. Examination of individual beta weights indicated that ED-General was not a significant predictor of depression symptoms on the DSM-Depression measure $(\beta=0.04, p=.642)$ or the BDI-SF measure $(\beta=-0.02, p=.820)$ and the $\Delta F$ from Model 2 to Model 3 was also not significant for DSM5-Depression $(p=.642)$ or BDI-SF $(p=.820)$. Contrary to our hypotheses, these results suggest that ED-General did not predict bipolar disorder mood symptoms.

We next examined whether the ED-Positive and ED-Negative subscales were predictors of symptom measures in Study 2. We also ran four separate regressions for each of the mania and depression symptom measures. Once again, the demographic variables in Block 1 did not predict any of the depression or mania measures (Model $\left.1 p \mathbf{s}^{>} .20\right)$. When PA and NA intensity were added in Block 2, the overall model was significant for depression symptoms on the DSM5-Depression measure (Model 2: $F_{(4,109)}=9.04, p<.001 ; R^{2}=.25, \Delta R^{2}=.23$ ) and the BDI-SF (Model 3: $F_{(4,109)}=19.59, p<.001 ; R^{2}=.42, \Delta R^{2}=.40$ ), as well as mania symptoms on the DSM5Mania measure (Model 3: $\left.F_{(4,109)}=2.50, p=.047 ; R^{2}=.08, \Delta R^{2}=.06\right)$. Specifically, NA intensity predicted increased depression symptom severity on the BDI-SF and difficulties associated with depression symptoms on the DSM5-Depression, as well as increased difficulties associated with 
mania symptoms on the DSM5-Mania measure. PA intensity also predicted increased mania symptoms on the ASRM measure. The overall models for Block 3 were significant for the two depression measures $(p s<.001)$, but not for the two mania measures $(p s>.07)$. However, the $\Delta F$ for the two depression measures was not significant $(p s>.70)$. Examination of individual beta weights suggested that neither ED-Positive nor ED-Negative were associated with depression symptoms $(p s>.50)$. Contrary to our hypotheses, neither ED-Positive nor ED-Negative valence scores predicted bipolar disorder mood symptoms.

\section{Study 2 Brief Discussion}

Contrary to Study 1, but in support of our hypotheses, Study 2 results suggested that EDGeneral was associated with increased trait bipolar risk (HPS-20). However, ED was not related to mania or depression mood symptom measures assessed within approximately 1 week prior to the ESM study. These results suggest that ED may be an important indicator of trait bipolar risk that may be contingent on various contextual factors, such as stress levels. The absence of associations between ED and mood symptoms could indicate that ED ability is less tied to current mood severity and more prominently influenced by trait levels of bipolar disorder risk tendencies. Although Study 2 improved upon Study 1 by collecting mania and depression symptoms more dimensionally and in a closer proximity to the experience-sampling study, mood symptoms are dynamic; thus, in future studies it will be critical to assess both concurrent and prospective predictive value of ED on bipolar disorder mood symptoms and trait bipolar risk, controlling for current mood state.

\section{General Discussion}

In the present investigation, we examined whether a dynamic emotion construct, ED, was associated with bipolar disorder risk across two distinct socioemotional contexts that were 
marked by varying degrees of acute external stressors (i.e., pre-COVID and during COVID) among emerging adults during their first year of college utilizing both state and trait levels of bipolar disorder risk measurement. Our hypotheses were partially supported. ED was associated with dimensions of bipolar risk, including state-level mood symptoms in Study 1, and trait-level dispositional bipolar risk in Study 2. Specifically, Study 1 results indicated that ED was not associated with trait bipolar risk. However, preliminary correlational analyses found that higher positive ED, but not negative or global ED was uniquely and significantly associated with increased mania symptom severity. Study 2 results suggested that global ED—but neither positive nor negative ED subscales alone — was linked to increased trait bipolar risk during a period of heightened stress co-occurring with the global COVID-19 pandemic, but not to bipolar disorder mood symptoms.

The results are consistent with past literature, however, this set of studies paints a more complex picture about the role of ED in the context of bipolar disorder risk than general trends in the existing literature suggest. To facilitate interpretation, we review differences between Study 1 (pre-COVID) and Study 2 (during COVID-19) that highlight the unique ways in which associations between ED and dimensions of bipolar risk may vary by context.

\section{Importance of Capturing Context in ED in Bipolar Disorder}

Although past ED research has examined clinically relevant outcomes, such as elevations in depression and anxiety symptoms, no study to our knowledge has examined associations between ED and more trait-like risk factors for psychological disorders. Importantly, we found that neither negative ED nor positive ED alone predicted trait bipolar risk as measured by the HPS-20. Rather, it was people with lower global emotion differentiation (including differentiation of positive and negative emotions) that had the highest dispositional traits 
characteristic of individuals at elevated risk for bipolar disorder. Researchers have theorized that ED is associated with adaptive psychological health outcomes via its link to emotion regulation (Kashdan et al., 2015). By providing more specific information about the type of emotions experienced, future work may help elucidate the role of ED in regulating emotions in bipolar mood difficulties.

We note that the findings that diverged across Study 1 and Study 2 raise important issues regarding the extent to which contextual factors may heavily influence the observed findings between ED and bipolar disorder relevant dimensions. We highlight key dimensions below that differentiate these two studies and highlight important methodological considerations for future work in bipolar disorder and psychopathology more generally when assessing ED. First, the two studies differed most markedly in the socioenvironmental context in which they took place. Study 1 occurred during a normative transition stressor to the first year of college and Study 2 occurred during an unanticipated, significant, and marked social and academic stressor during the acute outbreak and lockdown phase of the COVID-19 pandemic that resulted in an abrupt shift to remote learning, geographic relocation for many college students, and sudden severing of in-person social ties with college peers. Associations between lower ED and higher trait bipolar risk emerged only in the high stress context of Study 2 (i.e., during COVID). This suggests that experimental studies assessing ED in lower versus higher stress contexts may miss key links between ED and relevant psychological mood outcomes, perhaps due to lower variation in emotion experiences across the sampling period. Furthermore, it raises the question of whether ED ability varies within individuals across different socioenvironmental contexts.

We also note that the studies differed in the number of emotion items used to capture ED. Specifically, three additional emotion items (i.e., grateful/thankful, optimistic/hopeful, and 
lonely) were added to the existing scale of 9 total items to amplify the ability to capture variation in ED which may have bolstered our ability to detect links between ED and trait risk scores. Although the total number of emotion items that differed between the two studies was modest, these findings raise the possibility that detecting meaningful associations between ED and trait markers of mood risk may require a more nuanced assessment of ED than has previously been examined in prior literature (Thompson et al., 2021). It will also be critical to carefully tailor the specific emotion items assessed to the contextual setting in which the study is done (e.g., Study 2 added loneliness as a context-appropriate emotion during COVID-19) and emotions relevant to the theoretical models of affective processes driving a clinical phenomenon of interest (e.g., happy given its link to mania and depressed/blue to depression). Although this issue has not been explicitly raised to our knowledge in ED and clinical research, we suggest future studies carefully select ecologically-valid and feasible emotions.

We note that this investigation raises more general distinctions between how ED is conceptualized and operationalized in both studies here and previous literature. For example, ED was assessed by repeated experience-sampling ratings of current emotions, collapsed across multiple occasions over time, which arguably measures trait ED (Thompson et al., 2021). Yet some research has suggested that ED may vary over time, depending on developmental stage (e.g., Nook et al., 2018) or context (e.g., higher experiences of stress; Nook et al., 2020; Starr et al. 2019). Thus, future work is needed to map the diversity of the ways ED is operationalized with clinically relevant dimensions of mood difficulties in emerging adults.

\section{Limitations and Future Directions}

There are several important contributions of the present investigation. To our knowledge, this is the first series of studies to examine ED in the context of bipolar disorder risk. Another 
strength was the examination of both negative and positive ED measures, given prior work has largely focused on negative emotions specifically leaving a dearth of work examining the potential utility of positive ED. In light of the unique role of positive emotion disturbance in bipolar disorder and in numerous other psychopathologies (e.g., Gruber et al., 2020), greater attention to positive ED is essential to future research and could provide key insights into nuanced emotion experiences in diverse clinical phenomena.

Despite these notable strengths, there were several limitations of the present investigation which suggest directions for future research. First, although Study 2 was conducted amid the COVID-19 pandemic, which was presumed to be a naturally occurring socioemotional stressor, the experience-sampling procedure did not include daily measures of self-reported stress, which could have provided a rich opportunity to examine whether ED moderated links between stress and bipolar disorder-relevant symptoms. Second, while a major strength of the experiencesampling methodology was its ecological validity (i.e., the ability to measure ED in people's everyday lives), a drawback is that there was no standardization of emotion experiences across participants in their real-world lives and everyday contexts. Future work should include withinsubjects emotional experiences in daily life as well as more controlled laboratory assessments. Third, we used a short form of the bipolar disorder risk measure (HPS-20) to assess risk in two analogue and non-clinically diagnosed samples. While the HPS-20 is a valid predictor of bipolar spectrum disorders, it has been shown to capture more pathological features of hypomanic personality as compared to the original scale, and thus, may fail to detect potentially adaptive characteristics of hypomanic personality such as energetic and cheerful mood likely present in subclinical samples (Sperry et al., 2015). Future studies that examine comparisons between high versus low scorers on the full 48-item version of the HPS, or that oversample high scorers on the 
HPS, in addition to clinically diagnosed bipolar samples, are warranted. Lastly, the current studies were conducted with a relatively homogeneous sample of primarily white, financially privileged, American college students, which severely restricts the generalization of our study findings to more diverse socioenvironmental contexts. Future research should include historically marginalized populations who experience higher rates of chronic life stressors, such as poverty, discrimination, racism, food insecurity, and poorer physical and mental health outcomes. Furthermore, given the relative variation in how emotions are categorized and labeled within and across cultures and languages, expansion of ED research into more diverse populations and sociocultural contexts is important to accurately reflect the breadth and depth in which humans experience emotions (Villanueva et al., 2021).

Just as emotion models have emphasized the importance of individual context and goals in evaluating the adaptivity of a specific emotion in a given situation, the current studies indicate that links between ED and bipolar risk may vary by contexts. This work could improve our understanding of emotion functioning in affective disorders characterized by positive emotion disturbance and lead to insights on targets for risk identification and prevention efforts, as well as potential skills to integrate into psychotherapy. 


\section{Declaration of interest: none}

Role of funding source: This project was supported in part by a 2019 NARSAD Young Investigator Grant from the Brain \& Behavior Research Foundation (JG), start-up funds at the University of Colorado Boulder (JG), and the Office of Undergraduate Education at the University of Colorado Boulder. 


\section{References}

Alloy, L. B., Abramson, L. Y., Walshaw, P. D., Gerstein, R. K., Keyser, J. D., Whitehouse, W. G., Urosevic, S., Nusslock, R., Hogan, M. E., \& Harmon-Jones, E. (2009). Behavioral approach system (BAS) - relevant cognitive styles and bipolar spectrum disorders: Concurrent and prospective associations. Journal of Abnormal Psychology, 118(3), 459471. https://doi.org/10.1037/a0016604

Altman, E. G., Hedeker, D., Peterson, J. L., \& Davis, J. M. (1997). The Altman self-rating mania scale. Biological psychiatry, 42(10), 948-955. https://doi.org/10.1016/S00063223(96)00548-3

American Psychiatric Association (2013a). Diagnostic and statistical manual of mental disorders (5 $5^{\text {th }}$ ed.). Washington, DC: American Psychiatric Association.

American Psychiatric Association. (2013b). DSM-5 self-rated level 1 cross-cutting symptom measure-adult. Arlington, VA: American Psychiatric Publishing.

Anand, D., Chen, Y., Lindquist, K. A., \& Daughters, S. B. (2017). Emotion differentiation predicts likelihood of initial lapse following substance use treatment. Drug and Alcohol Dependence, 180, 439-444. https://doi.org/10.1016/j.drugalcdep.2017.09.007

Arnett, J. J. (2007). Emerging adulthood: What is it, and what is it good for? Child Development Perspectives, 1(2), 67-73. https://doi.org/10.1111/j.1750-8606.2007.00016.x

Auerbach, R.P., Alonso, J., Axinn, W.G., Cuijpers, P., Ebert, D.D., Greif Green, J., Hwang, I., Kessler, R.C., Liu, H., Mortier, P., Nock, M.K., Pinder-Amaker, S., Sampson, N.A., Aguilar-Gaxiola, S., Al-Hamzawi, A., Andrade, L.H., Benjet, C., Caldas-de-Almeida, J.M., Demyttenaere, K., ... \& Bruffaerts, R. (2016). Mental disorders among college 
students in the WHO World Mental Health Surveys. Psychological Medicine, 46(14), 2955-2970. http://doi.org/10.1017/S0033291716001665

Barrett, L. F. (1998). Discrete emotions or dimensions? The role of valence focus and arousal focus. Cognition and Emotion, 12, 579-599. https://doi.org/10.1080/026999398379574

Beck, A. T., \& Beck, R. W. (1972). Screening depressed patients in family practice: A rapid technic. Postgraduate Medicine, 52(6), 81-85. https://doi.org/10.1080/00325481.1972.11713319

Boden, M. T., Thompson, R. J., Dizén, M., Berenbaum, H., \& Baker, J. P. (2013). Are emotional clarity and emotion differentiation related?. Cognition and Emotion, 27(6), 961-978. https://doi.org/10.1080/02699931.2012.751899

Bruffaerts, R., Mortier, P., Kiekens, G., Auerbach, R. P., Cuijpers, P., Demyttenaere, K., Green, J. G., Nock, M. K., \& Kessler, R. C. (2018). Mental health problems in college freshmen: Prevalence and academic functioning. Journal of Affective Disorders, 225(1), 97-103. https://doi.org/10.1016/j.jad.2017.07.044

Cao, W., Fang, Z., Hou, G., Han, M., Xu, X., Dong, J., \& Zheng, J. (2020). The psychological impact of the COVID-19 epidemic on college students in China. Psychiatry Research, 287, 112934. https://doi.org/10.1016/j.psychres.2020.112934

Chandler, R. A., Wang, P. W., Ketter, T. A., \& Goodwin, G. M. (2008). A new US-UK diagnostic project: mood elevation and depression in first-year undergraduates at Oxford and Stanford universities. Acta Psychiatrica Scandinavica, 118(1), 81-85.

Demiralp, E., Thompson, R. J., Mata, J., Jaeggi, S.M., Buschkuehl, M., Barrett, L. F., Ellsworth, P. C., Demiralp, M., Hernandez-Garcia, L., Deldin, P. J., Gotlib, I. H., \& Jonides, J. 
(2012). Feeling blue or turquoise? Emotional differentiation in major depressive disorder. Psychological Science, 23(11), 1410-1416. https://doi.org/10.1177/0956797612444903

Dutra, S. J., West, T. V., Impett, E. A., Oveis, C., Kogan, A., Keltner, D., \& Gruber, J. (2014). Rose-colored glasses gone too far? Mania symptoms predict biased emotion experience and perception in couples. Motivation and Emotion, 38, 157-165. https://doi.org/10.1007/s11031-013-9363-4

Eckblad, M., \& Chapman, L. J. (1986). Development and validation of a scale for hypomanic personality. Journal of Abnormal Psychology, 95(3), 214-222. https://doi.org/10.1037/0021-843X.95.3.214

Erbas, Y., Ceulemans, E., Boonen, J., Noens, I., \& Kuppens, P. (2013). Emotion differentiation in autism spectrum disorder. Research in Autism Spectrum Disorders, 7(10), 1221-1227. https://doi.org/10.1016/j.rasd.2013.07.007

Fagiolini, A., Forgione, R., Maccari, M., Cuomo, a., Morana, B., Dell'Osso, M. C., Pellegrini, F., \& Rossi, A. (2013). Prevalence, chronicity, burden and borders in bipolar disorder. Journal of Affective Disorders, 148(2-3), 161-169. https://doi.org/10.1016/j.jad.2013.02.001

Feldman, L. A. (1995). Variations in the circumplex structure of emotion. Pers. Soc. Bull., 21, 806-817. https://doi.org/10.1177/0146167295218003

Furlanetto, L. M., Mendlowicz, M. V., \& Romildo Bueno, J. (2005). The validity of the Beck Depression Inventory-Short Form as a screening and diagnostic instrument for moderate and severe depression in medical inpatients. Journal of Affective Disorders, 86(1), 87-91. https://doi.org/10.1016/j.jad.2004.12.011 
Gross, J. J., \& Jazaieri, H. (2014). Emotion, emotion regulation, and psychopathology: An affective science perspective. Clinical Psychological Science, 2(4), 387-401. https://doi.org/10.1177/2167702614536164

Gruber, J. (2011). Can feeling too good be bad? Positive emotion persistence (PEP) in bipolar disorder. Current Directions in Psychological Science, 20, 217-221. https://doi.org/10.1177/0963721411414632

Gruber, J., Eidelman, P., Johnson, S. L., Smith, S., \& Harvey, A. G. (2011). Hooked on a feeling: Rumination about positive and negative emotion in inter-episode bipolar disorder. Journal of Abnormal Psychology, 120(4), 956-961. https://doi.org/10.1037/a0023667

Gruber, J., Harvey, A. G., \& Gross, J. J. (2012). When trying is not enough: Emotion regulation and the effort-success gap in bipolar disorder. Emotion, 12(5), 997-1003. https://doi.org/10.1037/a0026822

Gruber, J., Harvey, A. G., \& Johnson, S. L. (2009). Reflective and ruminative processing of positive emotional memories in bipolar disorder and healthy controls. Behaviour Research and Therapy, 47, 697-704. https://doi.org/10.1016/j.brat.2009.05.005

Gruber, J., Harvey, A. G., \& Purcell, A. (2011). What goes up can come down? A preliminary investigation of emotion reactivity and emotion recovery in bipolar disorder. Journal of Affective Disorders, 133, 456-466. https://doi.org/10.1016/j.jad.2011.05.009

Gruber, J., Johnson, S. L., Oveis, C., \& Keltner, D. (2008). Risk for mania and positive emotional responding: too much of a good thing? Emotion, 8(1), 23-33. https://doi.org/10.1037/1528-3542.8.1.23 
Gruber, J., Kogan, A., Mennin, D., \& Murray, G. (2013). Real-world emotion? An experiencesampling approach to emotion experience and regulation in bipolar I disorder. Journal of Abnormal Psychology, 122(4), 971-983. https://doi.org/10.1037/a0034425

Gruber, J., Villanueva, C., Burr, E., Purcell, J. R., \& Karoly, H. (2020). Understanding and taking stock of positive emotion disturbance. Social and Personality Psychology Compass, 14(1), 1-19. https://doi.org/10.1111/spc3.12515

Hair Jr, J. F., Hult, G. T. M., Ringle, C., \& Sarstedt, M. (2016). A primer on partial least squares structural equation modeling (PLS-SEM). Sage publications.

Johnson, S. L., Gruber, J. \& Eisner, L. R. (2007). Emotion and Bipolar Disorder. In J. Rottenberg \& S. L. Johnson (Eds.), Emotion and psychopathology: Bridging affective and clinical science (pp. 123-150). American Psychological Association. https://doi.org/10.1037/11562-006

Johnson, S. L., McKenzie, G., \& McMurrich, S. (2008). Ruminative responses to negative and positive affect among students diagnosed with bipolar disorder and major depressive disorder. Cognitive Therapy and Research, 32(5), 702-713. https://doi.org/10.1007/s10608-007-9158-6

Judd, L. L., Akiskal, H. S., Schettler, P. J., Endicott, J., Maser, J., Solomon, D. A., Leon, A. C., Rice, J. A., \& Keller, M. B. (2002). The long-term natural history of the weekly symptomatic status of bipolar I disorder. Archives of General Psychiatry, 59(6), 530-537. https://doi.org/10.1001/archpsyc.59.6.530

Kahneman, D., Krueger, A. B., Schkade, D. A., Schwarz, N., \& Stone, A. A. (2004). A survey method for characterizing daily life experience: The day reconstruction method. Science, 306(5702), 1776-1780. https://doi.org/10.1126/science.1103572 
Kalokerinos, E. K., Erbas, Y., Ceulemans, E., \& Kuppens, P. (2019). Differentiate to regulate: Low negative emotion differentiation is associated with ineffective emotion regulation use, but not strategy selection. Psychological Science, 8(5), 55. https://doi.org/10.1177/0956797619838763

Kashdan, T. B., Barrett, L. F., \& McKnight, P. E. (2015). Unpacking emotion differentiation: Transforming unpleasant experience by perceiving distinctions in negativity. Current Directions in Psychological Science, 24(1), 10-16. https://doi.org/10.1177/0963721414550708

Kashdan, T. B., \& Farmer, A. S. (2014). Differentiating emotions across contexts: Comparing adults with and without social anxiety disorder using random, social interaction, and daily experience sampling. Emotion, 14(3), 629-638. https://doi.org/10.1037/a0035796

Kashdan, T. B., Ferssizidis, P., Collins, R. L., \& Muraven, M. (2010). Emotion differentiation as resilience against excessive alcohol use: An ecological momentary assessment in underage social drinkers. Psychological Science, 21(9), 1341-1347. https://doi.org/10.1177/0956797610379863

Kennedy, N., Everitt, B., Boydell, J., Van Os, J., Jones, P. B., \& Murray, R. M. (2005). Incidence and distribution of first-episode mania by age: results from a 35-year study. Psychological Medicine, 35(6), 855-863. https://doi.org/10.1017/S0033291704003307

Kircanski, K., Lieberman, M. D., \& Craske, M. G. (2012). Feelings into words: contributions of language to exposure therapy. Psychological science, 23(10), 1086-1091. https://doi.org/10.1177/0956797612443830 
Kwapil, T. R., Miller, M. B., Zinser, M. C., Chapman, L. J., Chapman, J., \& Eckblad, M. (2000). A longitudinal study of high scorers on the hypomanic personality scale. Journal of Abnormal Psychology, 109(2), 222-226. https://doi.org/10.1037/0021-843X.109.2.222

Liu, D. Y., Gilbert, K. E., Thompson, R. J. (2019). Emotion differentiation moderates the effects of rumination on depression: A longitudinal study. Emotion. https://doi.org/10.1037/emo000062

Meads, D. M., \& Bentall, R. P. (2008). Rasch analysis and item reduction of the hypomanic personality scale. Personality and Individual Differences, 44(8), 1772-1783. https://doi.org/10.1016/j.paid.2008.02.009

Merikangas, K. R., Jin, R., He, J. P., Kessler, R. C., Lee, S., Sampson, N. A., Viana, M. C., Andrade, L. H., Hu, C., Karam, E. G., Ladea, M., Medina-Mora, M. E., Ono, Y. PosadaVilla, J., Sagar, R., Wells, J. E., \& Zarkov, Z. (2011). Prevalence and correlates of bipolar spectrum disorder in the world mental health survey initiative. Archives of General Psychiatry, 68(3), 241-251. https://doi.org/10.1001/archgenpsychiatry.2011.12

Meyer, T. D., \& Hautzinger, M. (2003). Screening for bipolar disorders using the Hypomanic Personality Scale. Journal of Affective Disorders, 75, 149-154. https://doi.org/10.1016/S0165-0327(02)00042-3

Myin-Germeys, I., Peeters, F. P. M. L., Havermans, R., Nicolson, N. A., DeVries, M. W., Delespaul, P. A. E. G., \& Van Os, J. (2003). Emotional reactivity to daily life stress in psychosis and affective disorder: An experience sampling study. Acta Pssychiatrica Scandinavica, 107(2), 124-131. https://doi.org/10.1034/j.1600-0447.2003.02025.x

Nook, E. C., Sasse, S. F., Lambert, H. K., McLaughlin, K. A., \& Somerville, L. H. (2018). The nonlinear development of emotion differentiation: Granular emotional experience is low 
in adolescence. Psychological Science, 29(8), 1346-1357.

https://doi.org/10.1177/0956797618773357

Nook, E. C., Flournoy, J. C., Rodman, A. M., Mair, P., \& McLaughlin, K. A. (2020). High emotion differentiation buffers against internalizing symptoms following exposure to stressful life events in adolescence: An intensive longitudinal study. Pre-print online manuscript. Retrieved from https://psyarxiv.com/q4uy8/

O’Toole, M. S., Jensen, M. B., Fentz, H. N., Zachariae, R., \& Hougaard, E. (2014). Emotion differentiation and emotion regulation in high and low socially anxious individuals: An experience-sampling study. Cognitive Therapy and Research, 38, 428-438. https://doi.org/10.1007/s10608-014-9611-2

Phillips, M. L., \& Vieta, E. (2007). Identifying functional neuroimaging biomarkers of bipolar disorder: toward DSM-V. Schizophrenia Bulletin, 33(4), 893-904. https://doi.org/10.1093/schbul/sbm060

Pond, R. S., Kashdan, T. B., DeWall, C. N., Savostyanova, A. L., Nathaniel, M., \& Fincham, F. D. (2012) Emotion differentiation moderates aggressive tendencies in angry people: A daily diary analysis. Emotion, 12, 326-337.

Selby, E. A., Wonderlich, S. A., Crosby, R. D., Engel, S. G., Panza, E., Mitchell, J. E., Crow, S. J., Peterson, C. B., \& Le Grange, D. (2014). Nothing tastes as good as thin feels: Low positive emotion differentiation and weight-loss activities in anorexia nervosa. Clinical Psychological Science, 2(4), 514-531. https://doi.org/10.1177/2167702613512794

Smidt, K. E., \& Suvak, M. K. (2015). A brief, but nuanced review of emotional granularity and emotion. differentiation research. Current Opinions in Psychology, 3, 48-51. 
Sperry, S. H., \& Kwapil, T. R. (2019). Affective dynamic in bipolar spectrum psychopathology: Modeling inertia, reactivity, variability, and instability in daily life. Journal of Affective Disorders, 25l(1), 195-204. https://doi.org/10.1016/j.jad.2019.01.053

Sperry, S. H., Walsh, M. A., \&. Kwapil, T. R. (2015). Measuring the validity and psychometric properties of a short form of the Hypomanic Personality Scale. Personality and Individual Differences, 82, 52-57. https://doi.org/10.1016/j.paid.2015.03.004

Starr, L. R., Hershenberg, R., Li, Y. I., \& Shaw, Z. A. (2017). When feelings lack precision: Low positive and negative emotion differentiation and depressive symptoms in daily life. Clinical Psychological Science, 5(4), 613-631. https://doi.org/10.1177/2167702617694657

Starr, L. R., Hershenberg, R., Shaw, Z. A., Li, Y. I., \& Santee, A. C., (2019). The perils of murky emotions: Emotion differentiation moderates the prospective relationship between naturalistic stress exposure and adolescent depression. Emotion, 20(6), 927-938. https://doi.org/10.1037/emo0000630

Suvak, M. K., Litz, B. T., Sloan, D. M., Zanarini, M. C., Barrett, L. F., \& Hofmann, S. G. (2011). Emotional granularity and borderline personality disorder. Journal of Abnormal Psychology, 120(2), 414-426. https://doi.org/10.1037/a0021808

Taquet, M. Quoidbach, J., Fried, E. I., \& Goodwin, G. M. (2021). Mood homeostasis before and during the coronavirus disease 2019 (COVID-19) lockdown among students in the Netherlands. JAMA psychiatry, 78(1), 110-112. https://doi.org/jamapsychiatry.2020.2389 Thompson, R. J., Springstein, T., \& Boden, M. (2021). Gaining clarity about emotion differentiation. Social and Personality Psychology Compass, e12584. https://doi.org/10.1111/spc3.12584 
Tomko, R. L., Lane, S., Pronove, L. M., Treloar, H. R., Brown, W. C., Solhan, M. B., Wood, P. K., \& Trull, T. J. (2015). Undifferentiated negative affect and impulsivity in borderline personality disorders: A momentary perspective. Journal of Abnormal Psychology, 124, 740-753. https://doi.org/10.1037/abn0000064

Tugade, M. M., Fredrickson, B. L., \& Feldman Barrett, L. (2004). Psychological resilience and positive emotional granularity: Examining the benefits of positive emotions on coping and health. Journal of Personality, 72(6), 1161-1190. https://doi.org/10.1111/j.14676494.2004.00294.x

Villanueva, C. M., Silton, R. L., Heller, W., Barch, D. M., \& Gruber, J. (2021). Change is on the horizon: call to action for the study of positive emotion and reward in psychopathology. Current Opinion in Behavioral Sciences, 39, 34-40. https://doi.org/10.1016/j.cobeha.2020.11.008

Wang, X., Hedge, S., Son, C., Keller, B., Smith, A., Sasogohar, F. (2020). Investigating mental health of US college students during the COVID-19 pandemic: Cross-sectional survey study. Journal of Medical Internet Research, 22(9), e22817. https://doi.org/10.2196/22817

Willroth, E. C., Flett, J. A. M., \& Mauss, I. B. (2019). Depressive symptoms and deficits in stress-reactive negative, positive, and within-emotion-category differentiation: A daily diary study. Journal of Personality, 1-11. https://doi.org/10.1111/jopy.12475

Zaki, L. F., Coifman, K. G., Rafaeli, E., Berenson, K. R., \& Downey, G. (2013). Emotion differentiation as a protective factor against nonsuicidal self-injury in borderline personality disorder. Behavior Therapy, 44(3), 529-540.

https://doi.org/10.1016/j.beth.2013.04.008 


\section{Footnotes}

${ }^{1}$ Recent studies that calculated ED as a sum score of positive emotion differentiation and negative emotion differentiation have found it explains unique variance above and beyond other measures of ED (Liu et al., 2019).

${ }^{2}$ Liu et al. (2019) hypothesized that positive ED may be uniquely important; however, the tendency for studies to include fewer positive emotion words may partially explain why several studies have not found any associations (Thompson et al., 2021).

${ }^{3}$ Prior to conducting analyses, survey responses were initially checked to exclude any obvious non-completers or ineligible responses consistent with previous approaches from this broader study protocol. For Study 1, an initial sample size of 1165 online survey responses were collected that included at least the first question on the survey (i.e., consent form). Survey responses were excluded if they fell into one of seven non-mutually exclusive categories: did not provide a required study identification number $(n=107)$, outside of the eligible age range $(n=124)$, not fluent in English $(n=35)$, not a first-year freshmen $(n=4)$, had duplicate survey responses $(n=92)$, did not complete the survey past the initial consent and demographic questions $(n=141)$, failed any of the attention check items $(n=221)$. We also checked whether any of the remaining participants had incomplete or inconsistent responding on the primary measure of trait bipolar risk (i.e., HPS-20), and ( $n=2)$ additional participants were excluded. We then excluded participants who completed $<50 \%$ of ESM survey prompts $(n=31)$.

${ }^{4}$ Theoretically, ICCs range from 0 to 1 . Participants with negative ICCs were changed to 0 before performing transformations and retained in analyses: Study $1(n=4)$; Study $2(n=5)$. This approach is consistent with past emotion differentiation studies (e.g., Boden et al., 2013; Liu et 
al., 2019). Per current recommendations (Thompson et al., 2021), we ran regression analyses excluding these participants and it did not change significant results in Study 1 or 2.

${ }^{5}$ Eligible study participants who had ICCs with zero variance on either positive emotions or negative emotions did not have reliable ED indices, and thus, were excluded from primary analyses: Study $1(n=23)$; Study $2(n=14)$. Zero variance was flagged by the SPSS warning: “scale has zero variance items". Further examination of these participants' ESM data indicated that there was little to no variability on negative or positive emotion items. Therefore, main analyses were run on a subset of participants who did not have emotion differentiation scores with a zero variance SPSS warning: Study $1(n=113)$; Study $2(n=122)$. In Study 2, when zero variance ICCs were included in analyses, ED-General (HPS-20: $\beta=-.14, p=.091$ ) was no longer a significant predictor of decreased trait bipolar disorder risk.

${ }^{6}$ Consistent with previous studies on bipolar risk and emotion functioning, we ran parallel analyses controlling for clinical symptoms. The main findings for Study 1 and Study 2 remained largely consistent when controlling for symptoms (See Supplementary Tables S1 and S2).

${ }^{7}$ For Study 2, an initial sample size of 1043 initial online survey responses were collected that included at least the first question on the survey (i.e., consent form). Similar to Study 1, survey responses were excluded if they did provide a required study identification number $(n=4)$, were outside the eligible age range $(n=5)$, were not fluent in English $(n=0)$, not a first-year student $(n=194)$, had duplicate survey responses $(n=120)$, did not complete the survey past the consent and demographic questions $(n=235)$, failed any of the attention check items $(n=340)$. Three additional participants were excluded $(n=3)$ who had incomplete or inconsistent responding on the primary measure of trait bipolar risk (i.e., HPS-20). We also excluded participants who completed $<50 \%$ of ESM survey prompts $(n=31)$. 
${ }^{8}$ Internal consistency for ASRM in Study 2 was low $(a=0.53)$, which could have been due to overall low variability on the scale as most participants scored at 0 or subthreshold.

${ }^{9}$ Based on Study 1 preliminary bivariate correlation findings, additional analyses were run in Study 2, with ED predicting state-level bipolar symptom measures using a subset of participants $(n=113)$ who completed symptom measures (i.e., DSM-Mania, ASRM, DSM5Depression, BDI-SF) during the Spring 2020 semester, an average of $M=7.56(\mathrm{SD}=6.25)$ days prior to the ESM data collection period. 
Table 1

Demographics and Clinical Characteristics Across Study 1 and Study 2 Eligible Participants

\begin{tabular}{|c|c|c|c|}
\hline & Study 1 & Study 2 & Statistic \\
\hline Age (Years) & $18.26(0.73)$ & $18.30(0.64)$ & $F=0.20$ \\
\hline $\begin{array}{l}\text { Male } \\
\text { Female } \\
\text { Other }\end{array}$ & $\begin{array}{c}36.0 \% \\
64.0 \% \\
0.0 \%\end{array}$ & $\begin{array}{l}20.7 \% \\
79.3 \% \\
0.7 \%\end{array}$ & $\chi^{2=} 7.78 * * *$ \\
\hline $\begin{array}{l}\text { Race/Ethnicity }(\mathrm{Y} / \mathrm{N}) \\
\text { Caucasian/White } \\
\text { African-American/Black } \\
\text { Asian-American/Pacific Islander } \\
\text { Hispanic/Latinx } \\
\text { Native American } \\
\text { Other }\end{array}$ & $\begin{array}{c}84.6 \% \\
2.2 \% \\
19.9 \% \\
5.1 \% \\
0.0 \% \\
0.7 \%\end{array}$ & $\begin{array}{c}77.9 \% \\
1.5 \% \\
18.4 \% \\
11.8 \% \\
2.2 \% \\
1.5 \%\end{array}$ & $\begin{aligned} \chi^{2} & =1.96 \\
\chi^{2} & =0.20 \\
\chi^{2} & =0.10 \\
\chi^{2} & =3.85 \\
\chi^{2} & =3.03 \\
\chi^{2} & =0.34\end{aligned}$ \\
\hline In a Current Relationship (vs. Not) & $40.4 \%$ & $37.5 \%$ & $\chi^{2}=0.25$ \\
\hline First-Generation College Status & $15.4 \%$ & $16.9 \%$ & $\chi^{2=} .11$ \\
\hline $\begin{array}{l}\text { Living Arrangement (Y/N) } \\
\text { Residence/Dorm Hall } \\
\text { Apartment Off campus } \\
\text { Alone } \\
\text { With Friend(s)/Roommate(s) } \\
\text { With Family Member(s) } \\
\text { Other }\end{array}$ & $\begin{array}{c}93.4 \% \\
2.9 \% \\
0.0 \% \\
6.6 \% \\
5.9 \% \\
1.5 \%\end{array}$ & $\begin{array}{c}89.7 \% \\
2.9 \% \\
1.5 \% \\
18.4 \% \\
5.9 \% \\
1.5 \%\end{array}$ & $\begin{array}{c}\chi^{2}=1.19 \\
\chi^{2}=.00 \\
\chi^{2}=2.02 \\
\chi^{2}=8.61 * * \\
\chi^{2}=.00 \\
\chi^{2}=.00\end{array}$ \\
\hline Socioeconomic Status Rating & $6.70(1.43)$ & $6.72(1.56)$ & $F=0.01$ \\
\hline HPS-20 & $7.40(3.83)$ & $8.01(3.77)$ & $F=1.80$ \\
\hline DSM5-Mania & $1.20(1.22)$ & $1.20(1.18)$ & $F=0.00$ \\
\hline ASRM & $5.79(3.51)$ & $1.12(1.62)$ & $F=191.53 * *$ \\
\hline DSM5-Depression & $1.58(1.14)$ & $2.04(1.16)$ & $F=9.75 * *$ \\
\hline BDI-SF & -- & $7.83(6.03)$ & -- \\
\hline
\end{tabular}

Note: Socioeconomic Ladder rated from 1 (people who are the worst off - those who have the least money, least education, and worst jobs or no job) and 10 (people who are the best off - those who have the most money, the most education, and best jobs). HPS-20=Hypomanic Personality Scale at study enrollment, 20-Item Version; DSM5-Mania=DSM-5 Cross-Cutting Symptom Measure Mania Maximum Score; ASRM=Altman Self Rating Mania scale at the time of the experience-sampling study (Spring 2019 for Study 1, Spring 2020 for Study 2); DSM5-

Depression=DSM-5 Cross-Cutting Symptom Measure Depression Maximum score; BDI-SF=Beck Depression Inventory - Short Form at the time of the experience-sampling study (Spring 2020 for Study 2). Race/Ethnicity and Living Arrangement options were six separate yes/no variables that were not mutually exclusive. ${ }^{*} p<.05$ comparing Study 1 and Study $2 ;{ }^{*} p<.01$ comparing Study 1 and Study 2 


\section{Table 2}

Bivariate Correlations Between Clinical and Experience-Sampling Study Measures in Study 1

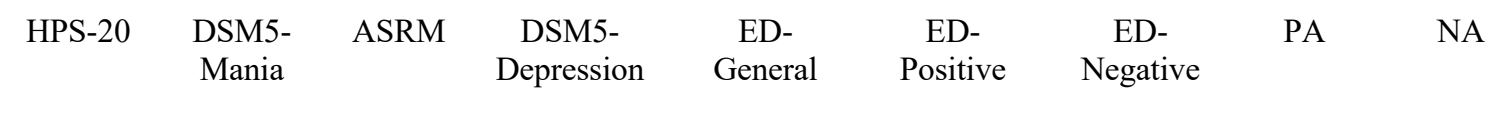

\begin{tabular}{|c|c|c|c|c|c|c|c|c|c|}
\hline HPS-20 & -- & $0.32 * *$ & 0.16 & 0.08 & -0.07 & -0.11 & -0.00 & 0.03 & 0.10 \\
\hline $\begin{array}{l}\text { DSM5- } \\
\text { Mania }\end{array}$ & & -- & $0.28 * *$ & 0.18 & -0.03 & -0.12 & 0.05 & 0.01 & 0.02 \\
\hline ASRM & & & -- & $-0.22 *$ & 0.13 & $0.24 *$ & 0.00 & $0.35^{* *}$ & -0.03 \\
\hline $\begin{array}{l}\text { DSM5- } \\
\text { Depression }\end{array}$ & & & & -- & -0.18 & -0.18 & -0.12 & $-0.35^{* *}$ & $0.45^{* *}$ \\
\hline ED-General & & & & & -- & $0.78^{* *}$ & $0.84^{* * *}$ & 0.17 & -0.30 ** \\
\hline ED-Positive & & & & & & -- & $0.31 * *$ & 0.14 & -0.04 \\
\hline $\begin{array}{l}\text { ED- } \\
\text { Negative }\end{array}$ & & & & & & & -- & 0.13 & $-0.43 * *$ \\
\hline PA & & & & & & & & -- & $-0.30 * *$ \\
\hline NA & & & & & & & & & -- \\
\hline
\end{tabular}

Note: HPS-20=20-item Hypomanic Personality Scale; DSM5-Mania=DSM-5 Cross-Cutting

Symptom Measure Mania Maximum Score; ASRM=Altman Self Rating Mania; DSM5-

Depression=DSM-5 Cross-Cutting Symptom Measure Depression Maximum Score;

$\mathrm{ED}=$ Emotion Differentiation; ED-General=General Emotion Differentiation (i.e., ED-Positive +

ED-Negative); ED-Positive=Positive Emotion Differentiation; ED-Negative=Negative Emotion

Differentiation; PA=Positive Affect; NA=Negative Affect. ${ }^{*} p<.05 ; * *<<.01$. 


\section{Table 3}

Regression Analyses using ED-General to Predict HPS-20 Scores

HPS-20

Study $1(n=113)$
HPS-20

Study $2(n=121)$

\begin{tabular}{|c|c|c|c|c|c|c|}
\hline Predictor & $\mathrm{R}^{2}$ & $\Delta \mathrm{R}^{2}$ & $\beta$ & $\mathrm{R}^{2}$ & $\Delta \mathrm{R}^{2}$ & $\beta$ \\
\hline Block 1 & .02 & .02 & & .01 & .01 & \\
\hline Age & & & .07 & & & .01 \\
\hline Gender & & & .11 & & & -.05 \\
\hline Block 2 & .04 & .02 & & $.13 * *$ & $.12 * *$ & \\
\hline PA & & & .07 & & & $.24 * *$ \\
\hline NA & & & .15 & & & $.23 *$ \\
\hline Block 3 & .04 & .00 & & $.16 * *$ & $.03 *$ & \\
\hline ED-General & & & -.00 & & & $-.19 *$ \\
\hline
\end{tabular}

Note: Regression analyses were conducted using a subset of eligible participants who had usable ED scores (see Footnote 5). HPS-20=20-item Hypomanic Personality Scale; Gender (Male=0, Female=1); PA=Positive Affect Mean Intensity; NA=Negative Affect Mean Intensity; $\mathrm{ED}=$ Emotion Differentiation; ED-General=General Emotion Differentiation (i.e., ED-Positive + ED-Negative); $\beta=$ Standardized beta coefficients from Model $3 ; * p<.05, * * p<.01$. 


\section{Table 4}

Regression Analyses Using ED-Positive and ED-Negative to Predict HPS-20 Scores

HPS-20

Study $1(n=113)$
HPS-20

Study $2(n=121)$

\begin{tabular}{|c|c|c|c|c|c|c|}
\hline Predictor & $\mathrm{R}^{2}$ & $\Delta \mathrm{R}^{2}$ & $\beta$ & $\mathrm{R}^{2}$ & $\Delta \mathrm{R}^{2}$ & $\beta$ \\
\hline Block 1 & .02 & .02 & & .01 & .01 & \\
\hline Age & & & .06 & & & -.01 \\
\hline Gender & & & .10 & & & -.05 \\
\hline Block 2 & .04 & .02 & & $.13 * *$ & $.12 * *$ & \\
\hline PA & & & .09 & & & $.24 * *$ \\
\hline NA & & & .17 & & & $.23^{*}$ \\
\hline Block 3 & .04 & .00 & & $.16 * *$ & .03 & \\
\hline ED-Positive & & & -.06 & & & -.10 \\
\hline ED-Negative & & & .06 & & & -.14 \\
\hline
\end{tabular}

Note: Regression analyses were conducted using a subset of eligible participants who had usable

ED scores (see Footnote 5). HPS-20=20-item Hypomanic Personality Scale; Gender (Male=0, Female=1); PA=Positive Affect Mean Intensity; NA=Negative Affect Mean Intensity; $\mathrm{ED}=$ Emotion Differentiation; ED-Positive=Positive Emotion Differentiation; EDNegative $=$ Negative Emotion Differentiation; $\beta=$ Standardized beta coefficients from Model 3; ${ }^{*} p<.05, * * p<.01$ 


\section{Table 5}

Bivariate Correlations Between Clinical and Experience-Sampling Study Measures in Study 2

\begin{tabular}{|c|c|c|c|c|c|c|c|c|c|c|}
\hline & HPS-20 & DSM5- & ASRM & DSM5- & BDI-SF & ED- & ED- & ED- & PA & NA \\
\hline & & Mania & & Depression & & General & Positive & Negative & & \\
\hline HPS-20 & -- & $0.28 * *$ & 0.13 & 0.16 & $0.21^{*}$ & $-0.23^{* *}$ & -0.15 & $-0.21 *$ & $0.26^{* *}$ & $0.20^{*}$ \\
\hline $\begin{array}{l}\text { DSM5- } \\
\text { Mania }\end{array}$ & & -- & $0.19 *$ & $0.18^{*}$ & 0.03 & -0.13 & -0.03 & $-0.18 *$ & 0.08 & 0.56 \\
\hline ASRM & & & -- & $-0.25^{* *}$ & $-0.22 * *$ & 0.02 & 0.07 & -0.06 & $0.24 * *$ & -0.08 \\
\hline $\begin{array}{l}\text { DSM5- } \\
\text { Depression }\end{array}$ & & & & -- & $0.65^{* *}$ & -0.08 & -0.01 & -0.42 & $-0.23 * *$ & $0.47 * *$ \\
\hline BDI-SF & & & & & -- & $-0.17^{*}$ & -0.07 & $-0.22 *$ & $-0.29 * *$ & $0.62^{* * *}$ \\
\hline ED-General & & & & & & -- & $0.79 * *$ & $0.84 * *$ & -0.07 & $-0.24 * *$ \\
\hline ED-Positive & & & & & & & - & $0.34 * *$ & -0.06 & -0.03 \\
\hline $\begin{array}{l}\text { ED- } \\
\text { Negative }\end{array}$ & & & & & & & & -- & -0.02 & $-0.35^{* *}$ \\
\hline PA & & & & & & & & & -- & -0.15 \\
\hline NA & & & & & & & & & & -- \\
\hline
\end{tabular}

Note: HPS-20=20-item Hypomanic Personality Scale; DSM5-Mania=DSM-5 Cross-Cutting Symptom Measure Mania Maximum Score; ASRM=Altman Self Rating Mania; DSM5-

Depression=DSM-5 Cross-Cutting Symptom Measure Depression Maximum Score; BDI$\mathrm{SF}=$ Beck Depression Inventory - Short Form; ED=Emotion Differentiation; EDGeneral=General Emotion Differentiation (i.e., ED-Positive + ED-Negative); EDPositive $=$ Positive Emotion Differentiation; ED-Negative=Negative Emotion Differentiation; $\mathrm{PA}=$ Positive Affect; $\mathrm{NA}=$ Negative Affect. ${ }^{*} p<.05 ; * * p<.01$. 